\title{
Corrigendum
}

\section{Corrigendum to "Prevalence of Corneal Astigmatism in Patients before Cataract Surgery in Western China"}

\author{
Wei Ma, ${ }^{1}$ Chengguo Zuo $\left(\mathbb{D},{ }^{1}\right.$ Weirong Chen, ${ }^{1}$ Shaoyang Zheng, ${ }^{1}$ Jiangang Xu, ${ }^{1}$ \\ Ruowen Gong, ${ }^{2}$ Maierhaba Mijiti, ${ }^{3}$ Kaidiliya Alifu, ${ }^{3}$ Lin Ding $\left(\mathbb{D},{ }^{3}\right.$ and Mingkai Lin $\mathbb{D}^{1}$ \\ ${ }^{1}$ State Key Laboratory of Ophthalmology, Zhongshan Ophthalmic Center, Sun Yat-Sen University, Guangzhou, China \\ ${ }^{2}$ Eye Institute, Eye and ENT Hospital, College of Medicine, Fudan University, Shanghai, China \\ ${ }^{3}$ People's Hospital of Xinjiang Urumqi Autonomous Region, Urumqi, China \\ Correspondence should be addressed to Lin Ding; dinglin85600@163.com and Mingkai Lin; linmk@mail.sysu.edu.cn \\ Received 18 November 2020; Accepted 18 November 2020; Published 18 January 2021 \\ Copyright (C) 2021 Wei Ma et al. This is an open access article distributed under the Creative Commons Attribution License, which \\ permits unrestricted use, distribution, and reproduction in any medium, provided the original work is properly cited.
}

In the article titled "Prevalence of Corneal Astigmatism in Patients before Cataract Surgery in Western China" [1], the contact details of Lin Ding were not included. The updated corresponding author information is shown above.

\section{References}

[1] W. Ma, C. Zuo, W. Chen et al., "Prevalence of corneal astigmatism in patients before cataract surgery in Western China," Journal of Ophthalmology, vol. 2020, Article ID 5063789, 6 pages, 2020. 